a combination of BS (bone scan) and CT (computerized tomography scan) is essential considering the unavailability of PET scan (positron emission tomography).

The aim of this study is to explore the bone involvement of gynecological cancer.

Methodology It is a retrospective study including gynecological cancer patients, referred for bone scan during 2019, at the Nuclear Medicine department of Tlemcen University Hospital in Algeria.

Two hours after the intravenous injection of $8-10 \mathrm{MBq} / \mathrm{kg}$ ${ }^{99 \mathrm{~m}}$ Tc-HMDP (hydroxy methylene diphosphonate), whole body scanning is accomplished by dual head hybrid gamma camera with low energy high resolution collimator.

Result(s)* Twenty-three bone scans were indicated in the initial staging of gynecological cancer patients, median age of 59 years [42-81], to explore bone pain in 15 cases $(65 \%)$ or an advanced stage of the neoplasms (peritoneal carcinomatosis, bone metastases or lung metastases) in 08 cases (35\%).

The different types of tumors are ovarian $(10 ; 43,5 \%)$, cervical $(9 ; 39,1 \%)$, and endometrial $(4 ; 17,4 \%)$.

The majority of BS (15 patients; 65,2\%) are relevant of osteoarthritis, involving both the spine and the peripheral joints, which are symptomatic in knees and shoulders. 4 patients $(17,4 \%)$ had normal scintigraphy.

Four patients $(17,4 \%)$ had multiple bone metastases with hot uptake scintigraphic pattern, involving both the axial and appendicular skeleton. Among these cases, the most common metastatic sites are pelvis, spine, and rib. Upon these metastatic patterns, 2 cases are related to endometrial cancer $(8,7 \%)$, the rest are related to cervical $(1 ; 4,34 \%)$ and ovarian carcinoma $(1 ; 4,34 \%)$. Bone pain was present in 3 metastatic cases involving spine, the rest was declared with bone metastases at CT.

Conclusion* Bone involvement of gynecological cancer is not frequent, it can be symptomatic or reported by CT scan. BS is a useful tool to explore with its good sensitivity the entire skeleton, in order to stage the neoplasm.

\section{PAP-SMEARS ALLOW THE IDENTIFICATION OF PROTEIN BIOMARKERS TO DIAGNOSE ENDOMETRIAL CANCER}

${ }^{1} S$ Cabrera*, ${ }^{2} E$ Coll-de la Rubia, ${ }^{3} E$ Martínez Garcia, ${ }^{3} \mathrm{~A}$ Lesur, ${ }^{4} \mathrm{~A}$ Reques, ${ }^{5} \mathrm{MA}$ Casares de Cal, ${ }^{5} \mathrm{~A}$ Gomez Tato, ${ }^{6} \mathrm{E}$ Sabidó, ${ }^{6} \mathrm{E}$ Borrás, ${ }^{7} \mathrm{R}$ Peiró, ${ }^{3} \mathrm{G}$ Dittmar, ${ }^{1} \mathrm{~A}$ Gil-Moreno, ${ }^{2} \mathrm{E}$ Colas. ${ }^{\prime} G y n e c o l o g i c$ Oncology Unit, University Hospital Vall d'Hebron, Vall d'Hebron Barcelona Hospital Campus and Autonomous University of Barcelona (UAB); ${ }^{2}$ Biomedical Research Group in Gynecology, Vall Hebron Institute of Research, Universitat Autònoma de Barcelona, CIBERONC, 08035 Barcelona, Spain; ${ }^{3}$ Quantitative Biology Unit, Luxembourg Institute of Health, L-1445 Strassen, Luxembourg; ${ }^{4}$ Pathology Department, Vall Hebron University Hospital, CIBERONC, 08035 Barcelona, Spain; ${ }^{5}$ University of Santiago de Compostela, Faculty of Mathematics, Santiago de Compostela, Spain; ${ }^{6}$ Center for Genomic Regulation, Proteomics, Barcelona, Spain; ${ }^{7}$ Hospital General de Catalunya, Sant Cugat del Vallés, Spain

\subsection{6/ijgc-2021-ESGO.105}

Introduction/Background* Endometrial cancer (EC) is the most common gynecological cancer in developed countries. There are no screening tools for its early diagnosis, and the diagnostic process starts with the apparition of related symptoms, mainly, abnormal vaginal bleeding (AVB). It is estimated that $\sim 7 \mathrm{M}$ women with AVB will undergo the diagnostic process every year in Europe, and from those, 9\% will have EC. Importantly, the current diagnostic process relies on the pathological examination of an endometrial biopsy that is always obtained by minimally-invasive to invasive methods. This overdiagnosis creates a big burden to the healthcare systems, so the development of non-invasive tools for EC diagnosis would revolutionize this scenario. Our aim is to approach a non-invasive diagnosis by the identification of protein biomarkers to accurately diagnose EC in liquid cervical cytologies.

Methodology The discovery phase consisted of a shotgun label-free proteomic approach. It included 60 patients $(20 \mathrm{EC}$, 20 controls suffering AVB without endometrial or cervical pathology, and 20 controls without endometrial pathology but cervical pathology). The levels of a statistically significant set of 75 proteins (110 peptides) from the discovery phase were measured in a verification phase including 234 (107 non-EC; 127 EC) patients by LC-MSMS/PRM. Analysis was performed using MaxQuant, Skyline, SPSS and R software.

Result(s)* The discovery study permitted to determine a total number of 2,888 proteins in our samples. Statistical analysis identified 75 potential proteins differently expressed between EC and non-EC patients to be further assessed and verified. Verification phase revealed the potential of 58 proteins measured in cervical cytologies to reach a non-invasive diagnosis of EC. Specifically, 16 proteins achieved an AUC $>0.75$, and 3 proteins an AUC $>0.8$. Additionally, an ELISA assay of the best performing protein was tested reproducing the results obtained by mass-spectrometry and reaching an $\mathrm{AUC}=0.927$ in this dataset.

Conclusion* We identified protein biomarkers in liquid cervical cytologies to diagnose EC patients with a diagnostic power up to $92 \%$. These results are promising for a subsequent development of an early and non-invasive screening tool for EC. This tool is expected to change the standard of care in EC diagnosis.

\section{7 EFFICACY OF TRANSVAGINAL AND TRANSABDOMINAL ULTRASOUND GUIDED BIOPSIES FOR HISTOLOGICAL DIAGNOSIS AND COMPLICATION RATES IN THE TWO BIOPSY METHODS}

B Hamid*, G Dadayal. Airedale General Hospital, Radiology department, Steeton, UK

\subsection{6/ijgc-2021-ESG0.106}

Introduction/Background* Thousands of women each year of varying age undergo Transvaginal (TV) and Transabdominal (TA) guided biopsy's due to suspected gynaecological cancers, the most common being Ovarian Cancer \& Primary Peritoneal Carcinomatosis. This study firstly looked at the efficiency and accuracy in attaining a conclusive histological diagnosis from a biopsy obtained via TA and TV method.

It also looked at whether there was any correlation between complications of TA and TV guided biopsies, and if so, were any confounding factors identified.

Methodology A search was done to identify all individuals who had undergone an ultrasound guided biopsy at Airedale General Hospital between March 2018 and August 2020. Those individuals who had undergone ultrasound guided biopsies at sites other than TA and TV were excluded from this study (e.g. axillary). 
Result(s)* A total of 28 individuals had undergone a TV or TA guided biopsy; 10 TV and 18 TA respectively, therefore these individuals were the focus of this study.

TV achieved a $100 \%$ success rate $(10 / 10)$ in obtaining a sample adequate for histological analysis, compared to TA which had a success rate of $89 \%(16 / 18)$.

9/10 samples obtained via TV route achieved a definitive histological diagnosis whereas 15/18 samples obtained via TA route achieved a definitive histological diagnosis.

3 TA guided biopsy's had to be repeated for differing reasons, compared to TV guided biopsy's which required no repeat sampling.

3 complications as a result of a biopsy were identified; in all cases this was bleeding. There was no clear association with bleeding and: size of needle used to obtain the biopsy, age of individual or method of biopsy.

Conclusion* TV guided biopsies were considerably more efficient, conclusive and representative of the clinical picture in obtaining a biopsy adequate for histological analysis, hence the reduced need for repeat biopsies.

Biopsies obtained via TV route were more successful at obtaining a definitive histological diagnosis compared to TA.

Bleeding was identified as the most common risk of a TV or TA guided biopsy but no confounding factors were found to correlate with this risk.

\section{A MACHINE LEARNING APPROACH APPLIED TO GYNECOLOGICAL ULTRASOUND TO PREDICT PROGRESSION-FREE SURVIVAL IN OVARIAN CANCER PATIENTS}

${ }^{1} \mathrm{~F}$ Arezzo*, ${ }^{1} \mathrm{~V}$ Loizzi, ${ }^{1} \mathrm{CM}$ Santarsiero, ${ }^{2} \mathrm{G}$ Cazzato, ${ }^{1} \mathrm{~V}$ Cataldo, 'M Mongelli, ${ }^{1} \mathrm{E}$ Cicinelli, ${ }^{1} \mathrm{G}$ Cormio. 'Obstetrics and Gynecology Unit, University of Bari; ${ }^{2}$ Pathology Section, University of Bari

\subsection{6/ijgc-2021-ESG0.107}

Introduction/Background* Ultrasound(US) is a cheap, non-invasive and well-recognized image modality for diagnosing and assessing ovarian cancer(OC). However, approximately $18 \%$ to $31 \%$ of adnexal lesions detected on US remain indeterminate. Machine learning(ML) is a promising tool for the implementation of complex multi-parametric algorithms. Despite the standardization of features capable of supporting the discrimination of ovarian masses into benign and malignant, there is the lack of accurate predictive modeling based on US examination for progression-free survival (PFS).

Methodology In this retrospective observational study, we analyzed patients with epithelial ovarian cancer(EOC) who were followed in a tertiary center from 2018 to 2019. Demographic, clinical and laboratory characteristics were collected as well as information about post-surgery histopathology. Furthermore, we recorded data about US examinations according to International Ovarian Tumor Analysis(IOTA) classification. Proper feature selection was used to determine an attribute core set. Random Forest(RFF) algorithm was trained and validated with 10-fold cross-validation to predict 12-month PFS. The accuracy of the algorithm was than assessed scoring accuracy and Area Under Receiver Operating Characteristic (AUROC).

Result(s)* Our analysis included n.32OC patients with mean age of $54.1 \pm 14.9$ years at diagnosis. Histotypes were n.19/32

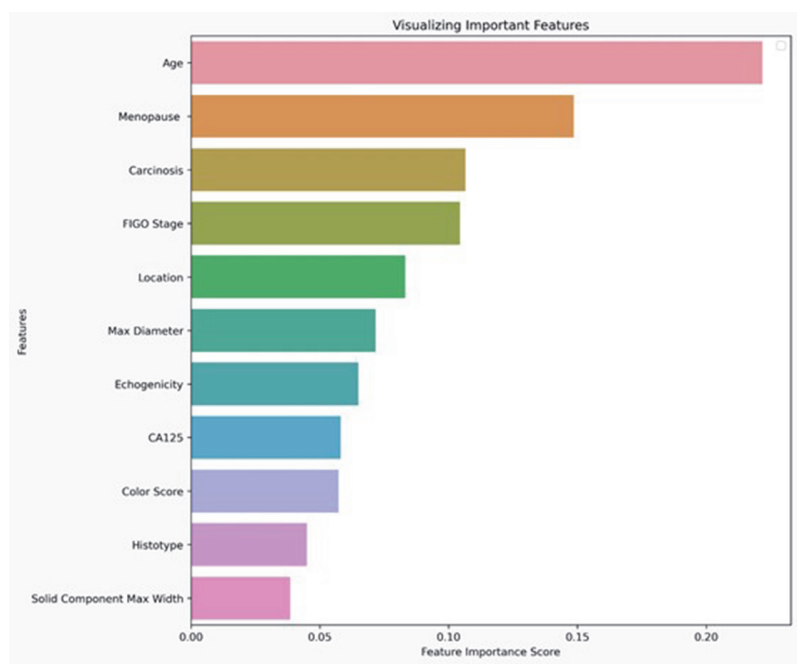

Abstract 954 Figure 1 Feature importance of the attribute coreset

(59.4\%) serous carcinoma, n.5/32(15.6\%) mucinous, n.5/32 (15.6\%) endometriod and n.3/32(9.4\%) clear cell. All patients underwent radical surgery. The attribute core set used to train machine learning algorithms is reported in figure 1. RFF showed an accuracy of 0.81 , AUROC 0.91 .

Conclusion* We developed an accurate model to predict 12month PFS in patients with OC based on a ML algorithm applied to gynecological ultrasound evaluation, requiring few easy-to-collect attributes.

\section{CAN SERUM HUMAN EPIDIDYMIS PROTEIN 4 (HE4) SUPPORT THE DECISION TO REFER A PATIENT WITH A PELVIC MASS TO AN ONCOLOGY CENTER?}

${ }^{1} \mathrm{P}$ Lof, ${ }^{1} \mathrm{R}$ Van de Vrie, ${ }^{2} \mathrm{~T}$ Korse, ${ }^{3} \mathrm{M}$ Van Gent, ${ }^{3} \mathrm{~S}$ Mom, ${ }^{4} \mathrm{~F}$ Rosier-van Dunné, ${ }^{5} \mathrm{M}$ Van Baal, ${ }^{6} \mathrm{H}$ Verhoeve, ${ }^{6} \mathrm{~B}$ Hermsen, ${ }^{7} \mathrm{M}$ Verbruggen, ${ }^{8} \mathrm{M}$ Hemelaar, ${ }^{8} \mathrm{~J}$ Van de Swaluw, ${ }^{9} \mathrm{H}$ Knipscheer, ${ }^{10} \mathrm{~J}$ Huirne, ${ }^{11} \mathrm{~S}$ Westenberg, ${ }^{12} \mathrm{~V}$ Van der Noort, ${ }^{1} \mathrm{~F}$ Amant, ${ }^{2} \mathrm{D}$ Van den Broek, ${ }^{1} \mathrm{C}$ Lok. ${ }^{1}$ Netherlands Cancer Institute, Center for Gynecologic Oncology Amsterdam, Amsterdam, Netherlands; ${ }^{2}$ Netherlands Cancer Institute, Department of Clinical Chemistry, Amsterdam, Netherlands; ${ }^{3}$ Amsterdam University Medical Center, Center for Gynecologic Oncology Amsterdam, Amsterdam, Netherlands; ${ }^{4}$ Tergooi Hospital , Department of Gynecology, Hilversum, Netherlands; ${ }^{5}$ Flevo Hospital, Department of Gynecology, Almere, Netherlands; ${ }^{6}$ olvg, Department of Gynecology, Amsterdam, Netherlands; 'Zaans Medical Center, Department of Gynecology, Zaandam, Netherlands; ${ }^{8}$ Dijklander Hospital, Department of Gynecology, Hoorn, Netherlands; ${ }^{9}$ Spaarne Hospital, Department of Gynecology, Haarlem, Netherlands; ${ }^{10}$ Amsterdam University Medical Center, Department of Gynecology, Amsterdam, Netherlands; " Noordwest Clinics, Department of Gynecology, Alkmaar, Netherlands; ${ }^{12}$ Netherlands Cancer Institute, Department of Statistics, Amsterdam, Netherlands

\subsection{6/ijgc-2021-ESG0.108}

Introduction/Background* Human epididymis protein (HE)4 has been shown to have a higher specificity than Cancer Antigen (CA) 125 in detecting ovarian cancer (OC). However, almost all studies are performed in oncology centers. As the prevalence of OC influences performance of predictive biomarkers, it is still unclear whether HE4 can be used to support referral decisions in general hospitals. We assessed the accuracy of HE4 with or without Risk of Malignancy Index (RMI) in differentiating benign from malignancy in patients with a pelvic mass from general hospitals. 